Mots. Les langages du politique

$71 \mid 2003$

Mondialisation(S)

\title{
Réactions aux comptes rendus du Dictionnaire d'analyse du discours
}

Patrick Charaudeau et Dominique Maingueneau

\section{(2) OpenEdition \\ Journals}

Édition électronique

URL : https://journals.openedition.org/mots/8703

DOI : $10.4000 /$ mots. 8703

ISSN : 1960-6001

Éditeur

ENS Éditions

Édition imprimée

Date de publication : 1 mars 2003

Pagination : 183-186

ISBN : 2-84788-027-5

ISSN : 0243-6450

Référence électronique

Patrick Charaudeau et Dominique Maingueneau, «Réactions aux comptes rendus du Dictionnaire d'analyse du discours », Mots. Les langages du politique [En ligne], 71 | 2003, mis en ligne le 06 mai 2008, consulté le 22 avril 2022. URL : http://journals.openedition.org/mots/8703 ; DOI : https://doi.org/ $10.4000 /$ mots.8703 
toriques sur l'évènementialité discursive, les recherches sur les pratiques langagières au travail, sur la transmission et la diffusion des savoirs, les terminologies, les discours médiatiques et communicationnels, l'argumentation, les discours constituants.

L'analyse du discours apparait bien ici comme un champ de recherches, partiellement structuré par quelques principes, partagés mais aussi discutés - notions-concepts de formation discursive, de contrat de communication, de genres et de types discursifs, de pratique langagière, constructivisme, références à Bakhtine, à Benveniste -, traversé par des courants de pensées, des méthodologies, des positions disciplinaires et interdisciplinaires diversifiées, tantôt complémentaires, tantôt carrément contradictoires. La démarche ouverte du $D A D$, extensive, autorise plus facilement des explorations et des articulations interdisciplinaires mais elle souligne davantage les absences: plusieurs options de linguistique textuelles, l'herméneutique, la pragmatique, la sociologie des langages, les approches logiques, sémiotiques, ou analytiques des textes, toute approches qui peuvent revendiquer leur place constitutive dans le champ.

L'analyse du discours ne trouve sans doute pas dans ces deux ouvrages l'acte (re)fondateur d'une discipline, une identité disciplinaire nettement affirmée, au sein des sciences du langage par exemple, mais elle y trouve à coup sûr une extension de son domaine, une certaine accumulation des expériences, de nouveaux contacts interdisciplinaires.

Pierre Fiala

\section{Réactions aux comptes rendus du Dictionnaire d'analyse du discours}

La critique est facile, mais l'art est difficile. C'est bien connu. Mais que serait l'art sans la critique? C'est là le caractère paradoxal et dialectique du rapport entre le discours et l'action. Il nous faut donc réagir, car c'est ainsi que doit s'instaurer le débat scientifique. Mais notre réaction doit tenir compte de certaines circonstances, car il est vrai qu'il n'y a pas de discours qui ne soit contraint. Nous ne sommes que les initiateurs, directeurs, coordinateurs d'un ouvrage dont l'essentiel de la nomenclature a été définie par les équipes qui ont collaboré, et dont chacun des auteurs (parmi lesquels nous nous comptons) a dit ce qui lui semblait pertinent dans le traitement de ses entrées. Responsables de l'ouvrage dont nous 
assumons les orientations, nous réagissons en réitérant les principes qui ont présidé à nos choix.

Comme l'a dit l'un des commentateurs, un dictionnaire «est un véritable terrain de chasse pour le spécialiste qui y pratique avec délectation la traque à l'erreur de fait, à la formulation obscure, à la nomenclature déficiente, au parti pris, au raccourci, à l'incohérence, à la contradiction, au cercle vicieux, aux simplifications et aux déformations ». Nous avions bien conscience de cela en nous lançant dans cette entreprise, d'autant plus qu'il ne s'agit pas d'un dictionnaire de langue, ni d'un lexique d'ouvrage spécialisé, ni d'un glossaire didactique. Il s'agissait de rassembler les concepts circulant dans un champ disciplinaire dont on sait qu'il est traversé par de multiples courants. Mais il fallait donner de la visibilité à une discipline en plein essor et qui est de plus en plus sollicitée par d'autres disciplines. Ainsi s'est imposée à nous cette évidence qu'il fallait se jeter à l'eau, avec tous les risques de noyade que cela comporte.

Deux types de problèmes se sont présentés que l'on retrouve d'ailleurs dans les commentaires dont on nous a fait part: 1. Notre position vis-à-vis de l'Analyse du discours comme discipline. 2. Le choix et le traitement des entrées.

1. Une discipline émerge à travers les interrogations qui surgissent lors de la pratique analysante, interrogations auxquelles on essaye d'apporter des réponses à la fois pratiques et théoriques, jusqu'à ce que se produisent des actes fondateurs sous la forme de textes qui deviennent textes de référence. Puis, la discipline se développe de confirmations en ruptures, de regroupements en diversifications théoriques et épistémologiques. Si donc il est normal de marquer l'histoire de l'AD par des moments fondateurs, il serait réducteur et inexact de la limiter à ces premiers gestes.

Le reproche majeur qui nous a été fait, et dont découlent certains autres, est que le $D A D$, dans la mesure où il chercherait à offrir des catégories stabilisées, liées à une "volonté de disciplinariser», dénaturerait l'AD: il «marginalise sa dimension critique initiale, en tant que lieu d'interrogation et d'expérimentation» (J. Guilhaumou). Ce reproche est parfaitement justifié, à condition évidemment d'admettre certains présupposés. En effet, il ne nous parait pas aller de soi que l'AD originelle fût foncièrement critique. À y regarder de plus près, l'AD «originelle» n'avait pas l'homogénéité qu'on lui prête parfois.

En France, dès ses débuts, elle a été partagée entre la problématique althussérienne, bien illustrée par Michel Pêcheux, et celle, plus souterraine, d'un Foucault. Or la dimension «critique» de l'archéologie foucal- 
dienne est loin d'aller de soi; du moins, elle exige une certaine lecture de son œuvre. Les premières recherches en AD en France n'avaient pas opté de manière aussi nette pour le caractère radicalement critique de leur démarche; on ne saurait sous-estimer la dimension catégorisante et descriptive, voire le caractère scientiste de certaines entreprises. L'idée même d'une «science de l'idéologie» s'accommodait mal d'une vision intégralement critique de l'AD. Il existait en France des démarches qui étaient fort éloignées du groupe de «l'École française», même en étendant cette désignation aux problématiques liées à Foucault.

L'AD n'est pas une affaire strictement française ou francophone; même s'il est indéniable qu'à ses débuts «l'École française» s'est développée dans une sorte d'insularité relative, on ne peut pas ou plus ignorer qu'il existe d'autres courants dans le monde, apparus également dans les années 1960, et qu'aujourd'hui les contacts sont incessants entre eux. C'est de cette diversité que cherche à prendre acte le $D A D$.

Il nous semble que le fonctionnement disciplinaire est une condition indépassable pour la recherche. Si on admet cela, il faut bien "outiller» l'AD, en particulier avec un dictionnaire, de la même manière qu'on «outille» la langue, pour reprendre une problématique de S. Auroux. Certes, l'AD doit maintenir une dimension critique (une dérive positiviste est toujours possible), mais l'AD ne peut pas être intégralement critique, sauf à sortir de l'orbite des sciences humaines et sociales, ce à quoi nous ne nous sentons pas prêts. Autre chose est d'affirmer que l'AD est traversée par une tension entre catégories et processus qui traversent leurs frontières, autre chose est d'affirmer que l'AD est de part en part critique. Le risque est de vouloir le beurre et l'argent du beurre, pour dire les choses trivialement. L'AD peut-elle construire un espace pour contester sa spatialité même, obéir et ne pas obéir aux contraintes des sciences humaines?

Nous pensons que l'AD tire sa force de la diversité et de l'opérativité des concepts qu'elle produit depuis bientôt une quarantaine d'années, concepts qui ont montré leur fécondité dans l'analyse de nombreux domaines (discours politique, médiatique, philosophique, didactique, scientifique...). C'est ainsi qu'elle contribue à alimenter une réflexion interdisciplinaire avec la philosophie, l'anthropologie sociale, la sociologie, la psychologie sociale, la rhétorique... à travers des problématiques aussi diverses que (l'interaction, l'imaginaire social, l'interdiscours, l'influence, l'argumentation, etc.).

2. Toute nomenclature de concepts dans les sciences humaines et sociales (mais pas seulement, car il en est de même entre biologie, étholo- 
gie, chimie, physique) se heurte au problème des frontières avec les disciplines connexes. Dans le cas de l'AD, s'ajoute celui de sa relation à la linguistique, son inévitable associé. Un dictionnaire d'AD doit-il être un surgeon de la linguistique ou doit-il s'en démarquer?

Notre position a été : rapport de dépendance et d'autonomie. De dépendance présupposée, car l'AD d'un certain point de vue n'a été possible que grâce à la rupture que la linguistique a produite vis-à-vis de la tradition philologique et grammaticale, et que l'AD hérite de bien de ses aspects méthodologiques et conceptuels, et se nourrit constamment de ses apports; d'autonomie parce que l'AD a fini par se construire son propre objet, ses propres outils, sa propre théorisation.

Les remarques de $\mathrm{B}$. Pottier et $\mathrm{P}$. Fiala soulignent à juste titre le problème des frontières, interne et externe, que cela ne manque pas de poser, B. Pottier insistant plutôt sur l'absence de termes de linguistique et P. Fiala sur celle de termes des sciences humaines et sociales. Notre choix a été de trouver une voie moyenne entre ces deux précipices: intégrer la totalité des termes de la linguistique, intégrer la totalité du vocabulaire des sciences humaines. Nous avons privilégié ceux qui de part et d'autre nous semblaient indispensables et ne figuraient pas déjà dans les dictionnaires d'autres disciplines. On ne pouvait pas faire plusieurs dictionnaires en un, au risque de voir se dissoudre la spécificité de l'AD. Il fallut faire des choix, douloureux parfois, et nous sommes conscients des insuffisances que ceux-là ont pu produire. Il en est de même pour le traitement des articles, pour lequel nous avons demandé aux auteurs d'associer un inventaire des usages du terme et une perspective organisatrice personnelle. Aussi la remarque de M.-A. Paveau à propos de «Doxa» est-elle juste, mais à la décharge de l'auteur, il faut remarquer qu'il avait pour mission de traiter cette notion dans le seul cadre de l'argumentation.

Notre intention était de circonscrire un champ original, d'inscrire les notions dans des réseaux conceptuels cohérents et d'orienter le lecteur dans une perspective discursive. Ainsi nous espérons que ce dictionnaire aide non seulement à comprendre les travaux existants mais encore à développer des recherches nouvelles. 